Rev. Elev. Med. vét. Pays trop., 1975, 28 (4) : 473.479

\title{
Parabronema longispiculatum n. sp. parasite stomacal de l'éléphant d'Afrique (Loxodonta africana Blumenbach, 1797)
}

\author{
par M. GRABER (*)
}

\begin{abstract}
RESUME
L'auteur, à partir d'exemplaires recueillis dans l'estomac de deux Eléphants abattus au Tchad, décrit un nouveau Spiruridé, Parabronema longispiculaium que ses caractères morphologiques et ses dimensions permettent de distinguer d'une espèce voisine, également parasite stomacal de l'Eléphant d'Afrique, Parabronema rhodesiense YORKE et MAPLESTONE, 1926.
\end{abstract}

\section{INTRODUCTION}

A l'occasion de battues effectuées en 1969 près de N'Djamena (Fort-Lamy), dans les régions de Koundoul et de Gnamre (Préfecture du Chari-Baguirmi, République du Tchad), deux Eléphants (Loxodonta africana BLUMENBACH, 1797) ont été tués et autopsiés.

Dans l'estomac, ont été isolés des Parabronema dont les caractères morphologiques et les lésions qu'ils provoquent méritent de retenir l'attention.

\section{MATERIEL ET METHODE}

Au total, deux cent quatre vingt douze Parabronema ont été recueillis, soit soixante quatorze parasites ( 31 mâles et 43 femelles) dans l'estomac du premier animal et deux cent dix-huit (51 mâles et 167 femelles) chez le second.

Vingt mâles et vingt-cinq femelles ont été éclaircis au lacto-phénol, mesurés et dessinés.

En outre, au cours des deux autopsies, les lésions stomacales ont été soigneusement observées.

(*) Chaire de Parasitologie. Ecole Nationale Vétérinaire. 69337 Lyon Cedex I.

\section{RESULTATS}

\section{Le parasite}

\section{I.1. Description}

Les Parabronema sont des Spirurida appartenant, selon la nouvelle classification proposée par CHABAUD (6), à la famille des Habronematidae, sous-famille des Parabronematinae.

Ce sont des Nématodes de petite taille, à cuticule striée, dont les extrémités vont en s'amincissant, surtout dans la région antérieure. Celleci, en forme de cône, présente une structure complexe caractéristique du genre Parabronema (3). La cuticule se plisse fortement en donnant naissance à six appendices ou "cordons » répartis en deux systèmes, l'un antérieur, l'autre postérieur (planche I). Ceux du système postérieur sont en forme de fer à cheval, comprimé latéralement, et se terminent à $132-168 \mu$ de l'extrémité céphalique (tableau $n^{*} \mathrm{I}$ ). La tête, par ailleurs, porte une bouche entourée de pseudo-lèvres et de lèvres sur lesquelles s'insèrent quatre papilles latéro-médianes et quatre papilles médico-médianes.

L'ouverture buccale, limitée par des dents, débouche dans un vestibule cylindrique à parois épaisses dont les dimensions sont données au tableau $\mathbf{n}^{\circ} \mathrm{I}$. 


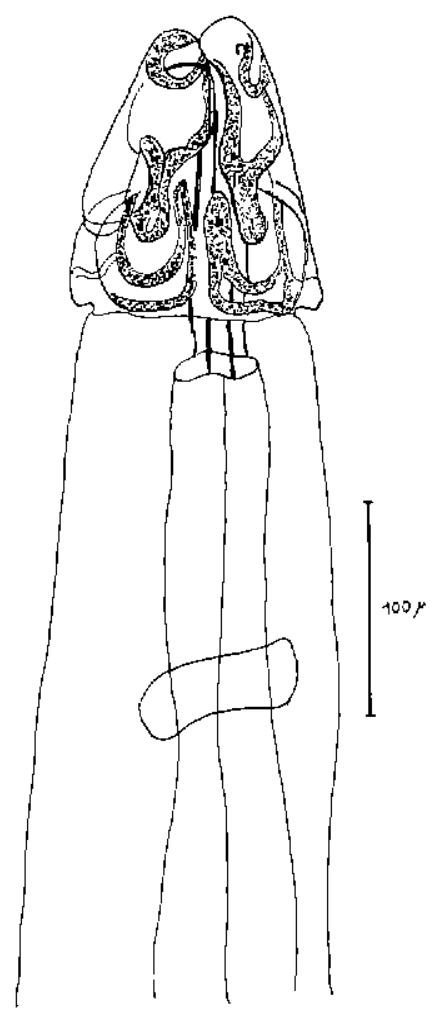

Planche I.

Parabronema rhodesiense longispiculatum. Extrémité antérieure.
Lui succède un œsophage divisé en deux parties, une partie antérieure musculaire et une partie postérieure glandulaire, beaucoup plus longue et beaucoup plus large. L'anneau nerveux se trouve placé à $250-320 \mu$ de l'apex (planche I). Déirides et pore excréteur sont un peu en arrière de l'anneau nerveux.

Il n'existe pas d'ailes latérales.

La femelle mesure 10,5 à $13,2 \mathrm{~mm}$ de long sur $307-420 \mu$ de large. La vulve est située dans la région antérieure du corps, au voisinage de la terminaison de l'œsophage. La distance entrè cet organe et l'ouverture vulyaire varie dans des proportions sensibles et semble dépendre de l'état de maturité sexuelle du parasite: 60 à $304 \mu$ chez des femelles bourrées d'embryons, $720 \mu$ chez une femelle immature.

L'ovojecteur, étroit et allongé, est pourvu d'un sphincter en S (planche III, figure 2). Ovaires et utérus ont la disposition classique des Parabronema (3, 5, 17).

La queue est courte, légèrement recourbée (planche III, fig. 1) et sa pointe est surmontée d'un petit bouton plus ou moins visible.

Les femelles sont vivipares et, dans l'utérus, s'accumulent des embryons de $48-54 \mu$, repliés

TABL. $\mathrm{N}^{\circ} \mathrm{I}$-Dimensions comparées de Parabronema rhodesiense et de Parabronema Iongispiaulatum

\begin{tabular}{|c|c|c|c|c|}
\hline & \multicolumn{2}{|c|}{ Parabronema rhodesiense Zongispiculatum } & \multicolumn{2}{|c|}{ Earabronema rhodesiense } \\
\hline & Mâle (20) & Femel1e (25) & Mâle & Femelle \\
\hline Longueur (en $\mathrm{nm}$ ) & $6,3-7,9\left(6,95^{+}\right)$ & $10,5-13,2\left(11,7^{+}\right)$ & $7-8$ & $9-10$ \\
\hline Largeur (en $\mu$ ) & $236-307(275,7)$ & $307-420(376,6)$ & - & - \\
\hline Largeur de la tête ( $(\mu)$ & $114-126(121,6)$ & $128-150(136,3)$ & - & - \\
\hline $\begin{array}{l}\text { Distance des cordons postêrieurs } \\
\text { à l'extrémité antêrieure }(\mu)\end{array}$ & $132-150(151)$ & $140-168(149,1)$ & 115 & $130-140$ \\
\hline Vestibule $(\mu)$ & $156-174(165,1)$ & $160-204(176,5)$ & 250 & 175 \\
\hline Distance a $I^{\dagger}$ extrëritể antérieure & & & & \\
\hline - de l'anneau nerveux $(\mu)$ & $250-312(278,4)$ & $250-320(287,4)$ & - & - \\
\hline - du pore excréteur $(\mu)$ & $324-372(346,3)$ & $348-420(364,7)$ & - & - \\
\hline Longueur de I'oesophage (am) & $1,6-1,8 \quad(1,7)$ & $1,8-2,18(1,97)$ & 1,03 & 1,23 \\
\hline Stries cuticulaires $(\mu)$ & 436 & 5 à 8 & - & - \\
\hline Spicules & & & & \\
\hline - droit $(\mu)$ & $318-371(344,4)$ & - & 330 & - \\
\hline - gauche (mm) & $1,18-1,52(1,32)$ & - & 612 & - \\
\hline Cubernaculum $(\mu)$ & $36-54(48,2)$ & - & - & - \\
\hline Longueur crêtes caudales (nm) & $1,06-1,32(1,19)$ & - & - & - \\
\hline Longueur de la queue $(\mu)$ & - & $247-318(282,7)$ & - & 200 \\
\hline $\begin{array}{l}\text { Distance de la vulve à l'extré- } \\
\text { mité postérieure de l'oesophage }(\mu)\end{array}$ & - & $60-340(144,2)$ & - & $860-900$ \\
\hline Embryons $(\mu)$ & - & $48-54$ & - & \\
\hline
\end{tabular}

+ Les chuffres entre parenthèses indiquent les moyennes. 


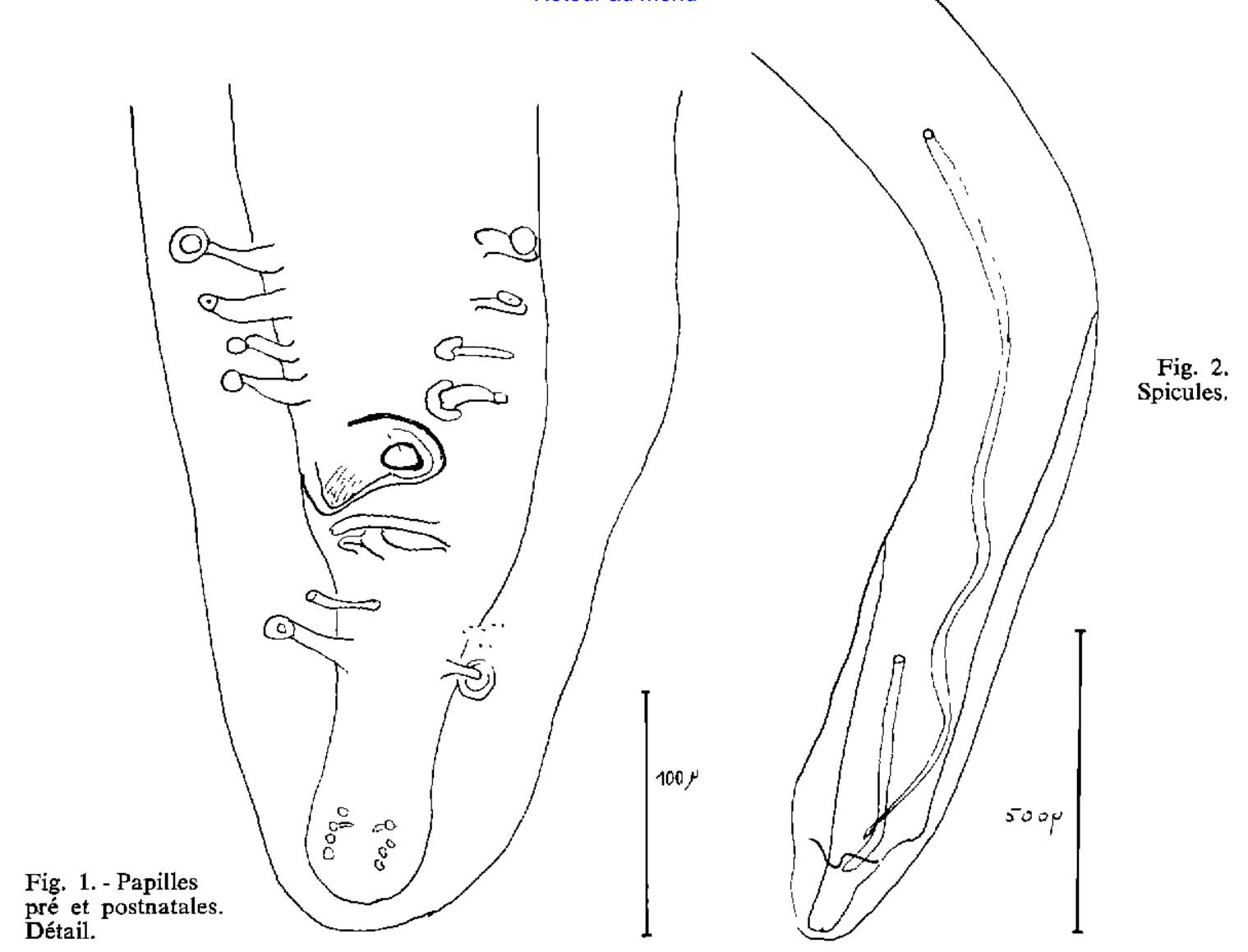

Planche II. - Extrémité postérieure d'un mâle de Parabronema rhodesiense longispiculatum.

Fig. 1. - Queue.
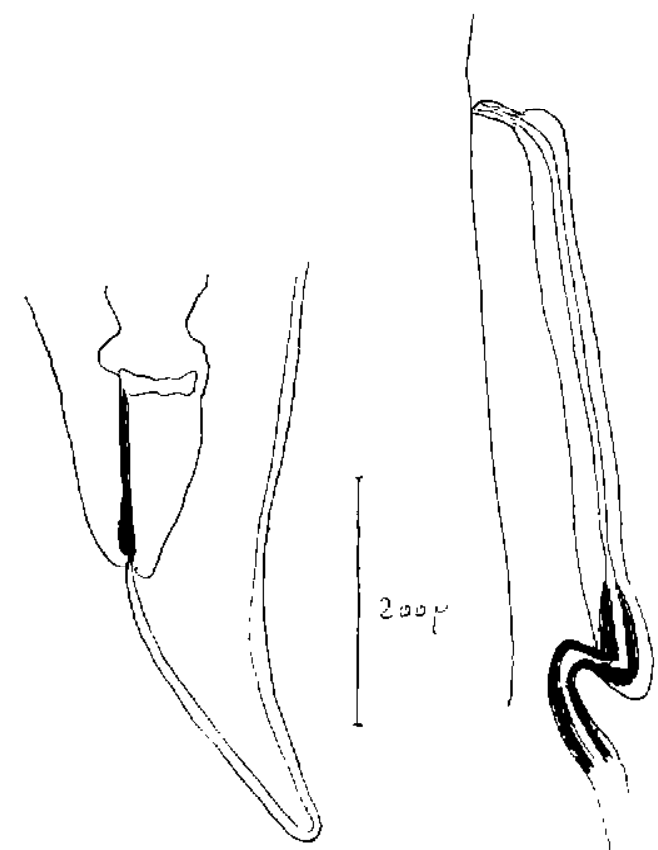

Fig. 2. - Ovojecteur.

Planche III. - Femelle de Parabronema rhodesiense longispiculatum. 
sur eux-mêmes et entourés d'une fine membrane.

Le mâle est moins long que la femelle (tableau $\mathrm{n}^{\circ} \mathrm{I}$ ). La queue, obtuse, est soutenue par deux faibles ailes latérales (planche II, figure 2). Elle s'enroule sur elle-même en spirale. Sa face ventrale, sur une longueur de $1,2 \mathrm{~mm}$ en moyenne, est couverte de stries longitudinales formant de véritables crêtes. Elle porte, en outre, plusieurs séries de papilles (planche II, figure I) :

- Quatre paires préanales.

- Deux paires post-anales. Les papilles de la paire la plus antérieure sont rejetées sur le plan médian et dissymétriques; la papille gauche est, en effet, plus proche du cloaque que celle de droite.

- Un groupe terminal comprenant quatre paires de très petites papilles et les phasmides.

- Une papille double est plaquée immédiatement en avant du cloaque : elle n'est pas toujours facile à observer.

Les spicules sont inégaux et dissemblables (planche II, figure 2). Le spicule gauche, très mince et à extrémité effilée, est presque quatre fois plus long que le spicule droit. Celui-ci, large et trapu, se termine en pointe mousse.

\section{I.2. Discussion}

On dénombre actuellement dix espèces de Parabronema, parasites de divers Mammifères domestiques et sauvages de l'ancien et du nouveau monde.

I.2.1. Quatre d'entre elles sont des Nématodes de grande taille dépassant $10 \mathrm{~mm}$ chez le mâle et $20 \mathrm{~mm}$ chez la femelle. La vulve est très éloignée de l'extrémité antérieure (plus de 4,3 $\mathrm{mm}$ ) et l'ornementation céphalique est relativement simple. Les spicules sont ou plus longs, ou plus courts que ceux des Nématodes de l'Eléphant du Tchad. Il s'agit de :

- Parabronema bonnei VAN THIEL, 1925 découvert dans l'intestin d'un Singe hurleur du Surinam.

- Parabronema okapi (*) LEIPER, 1935, parasite de l'Okapi au Zaïre (4) et dans divers jardins zoologiques d'Europe (I).

- Parabronema skrjabini RASSOWSKA, 1924 qui affecte, en Afrique, I'Okapi (I), la Girafe, le Buffle, l'Hippotrague, l'Ibex de Nubie

(*) Dans le sens où l'entend BAER (I).
(28), le Dromadaire (10) et en Asie (Turkestan, Indes), le Chameau, le Dromadaire, le Bœuf, le Mouton et la Chèvre (21).

- La quatrième espèce, Parabronema africanum BAYLIS, 1921 est propre à l'Eléphant d'Afrique et a été recueillie au Zoo de Londres (3), en République démocratique du Congo, au Cameroun (5), au Liberia, au Zaïre (30) et en Afrique du sud (2). Le spicule gauche est très long $(3,3$ à $6,3 \mathrm{~mm})$.

I.2.2. Deux autres espèces ne sont connues que par leurs femelles :

- Parabronema pecariae IVASHKIN, 1960 chez un Pécari du Texas;

- Parabronema rhinocerotis décrit par KHALIL (18) à partir d'une seule femelle provenant de l'intestin d'un Rhinocéros abattu en Ethiopie. à proximité de la rivière Ousmée (20).

Parabronema rhinocerotis est proche du $\mathrm{Pa}$ rabronema de l'Eléphant du Tchad, ce qui n'est guère étonnant, compte tenu du fait que les Nématodes de l'Eléphant se retrouvent fréquemment chez les Rhinocéros et réciproquement. Cependant, l'absence de mâle empêche de tirer des conclusions définitives.

1.2.3. Parabronema smithii COBBOLD, 1882, parasite stomacal de l'Eléphant d'Asie (Elephas maximus), est un très petit Nématode ( $4 \mathrm{~mm}$ chez le mâle; $8 \mathrm{~mm}$ chez la femelle). Les spicules sont courts, surtout le gauche $(560 \mu)$.

I.2.4. Parabronema indicum BAYLIS, 1921 de l'Eléphant d'Asie et Parabronema roundi FITZSIMMONS, 1962 du Rhinocéros africain ont à peu près la même taille que le Parabrone$m a$ du Tchad (7,9-8,4 mm chez le mâle; 11,4$13,6 \mathrm{~mm}$ chez la femelle). Dans les deux cas, les spicules sont moins longs et la distance de la. vulve à l'extrémité antérieure supérieure. De plus, le vestibule de Parabronema roundi mesure 192-220 $\mu$ (mâle) et 232-240 $\mu$ (femelle). Dans cette espèce, les déirides sont situées bien plus en avant, au niveau de la jonction vestibulocesophagienne.

I.2.5. L'espèce avec laquelle le Parabronema du Tchad a le plus d'affinité est Parabronema rhodesiense, décrit brièvement en 1926 par YORKE et MAPLESTONE à partir d'exemplaires prélevés dans l'estomac d'un Eléphant tué en Rhodésie (?), et revu depuis par Condy (9) dans le même pays et sur le même animal. 
Toutefois, la lecture du Tableau $\mathrm{n}^{0}$ I montre qu'il existe entre les deux Nématodes de sensibles différences :

- L'œsophage et le spicule gauche des Parabronema du Tchad sont beaucoup plus longs;

- La vulve est placée plus près de la terminaison de l'oesophage;

- Chez la femelle, la longueur de la queue varie selon les individus. En moyenne, elle dépasse de $80 \mu$ celle de Parabronema rhodesiense;

- La première papille post-cloacale gauche de Parabronema rhodesiense est plus éloignée du cloaque.

Dans ces conditions, il nous a paru opportun de proposer la création d'une nouvelle espèce, Parabronema longispiculatum.

\section{Les lésions}

Les Parabronema vivent en général dans l'estomac de leur hôte, exceptionnellement dans le foie et le cour $(5 ; 22)$.

Les lésions qu'ils provoquent sont de deux types:

II.1. Sur la grande courbure stomacale, apparaissent des plages enflammées et boursouflées et, surtout, des ulcères plus ou moins nombreux, pouvant atteindre 4 à $5 \mathrm{~cm}$ de diamètre. Leurs bords relevés délimitent de petites cuvettes recouvertes de débris néocrotiques jaunâtres, au milieu desquels sont logés les Parabronema. La plupart s'enfoncent dans le tissu de nécrose. Cependant, certains d'entre eux peuvent se trouver libres à la surface de la muqueuse, en dehors de toute lésion : ils sont alors englobés dans une épaisse couche de mucus.

$\mathrm{Ce}$ sont essentiellement les cryptes stomacales qui sont envahies. Parfois, les parasites gagnent la muqueuse ou la sous-muqueuse.

L'organe réagit. On note la présence de nombreux éosinophiles et neutrophiles et, en profondeur, de plasmocytes et de lymphocytes. Progressivement, se forme un tissu de substitution de type granuleux (2).

Des lésions de cette nature ont été mises en évidence dans l'estomac de l'Eléphant $\mathrm{n}^{\circ}$ I (Koundoul). Elles semblent assez fréquentes, tant chez l'Eléphant d'Asie porteur de Parabronema smithii (24) que chez l'Eléphant
d'Afrique : BASSON et Collab. (2) les ont rencontrées chez quinze des vingt-deux animaux sacrifiés au Parc National Kruger en Afrique du Sud, le parasite en cause étant, dans ce cas, Parabronema africanum.

II.2. Chez le second Eléphant (Gnamre), les lésions ont un tout autre aspect : les ulcères font place à des nodules, véritables pseudo-tumeurs, de taille variable, de consistance ferme et creusés de multiples cavités communiquant avec l'extérieur. Celles-ci renferment les Parabronema enfouis dans une matière grisâtre, purulente et nauséabonde.

Des observations semblables ont été faites à différentes reprises, principalement chez l'Eléphant d'Asie et des nodules ont été trouvés dans la paroi stomacale de Proboscidiens autopsiés en Australie (24) (*), en Allemagne au Zoo de Berlin (9) et, plus anciennement à Londres (8) (*).

Les lésions ulcéreuses de Parabronémose ressemblent à celles que l'on observe dans l'estomac des chevaux atteints d'habronémose à $\mathrm{Ha}$ bronema muscae ou à Habronema microstomum et les lésions nodulaires à celles de Draschia megastoma.

Il existe, en effet, une parenté biologique étroite entre tous ces Spiruridés. On connaît, depuis les travaux d'IVASHKIN $(14,15)$, le cycle évolutif de Parabronema skrjabini. L'hôte intermédiaire est un Muscidae, de la sous-famille des Stomoxynae, Lyperosia titillans qui pond ses cufs dans les matières fécales fraîches des ruminants. Il en sort des larves qui, dans les mêmes excréments, absorbent les œufs de Parabronema.

Ceux-ci évoluent parallèlement aux métamorphoses que subit l'insecte: Larve $\mathbf{L}_{1}$ chez la larve de Lyperosia, Larve $\mathbf{L}_{2}$ chez la pupe, Larve $L_{3}$ chez l'imago. Les larves $L_{3}$ infestantes, obtenues au bout de $15-18$ jours à la température de $20-28^{\circ} \mathrm{C}$., se rassemblent dans le labium du Lyperosia parvenu au stade imaginal et perforent la membrane labellaire, lorsque l'insecte se pose sur les muqueuses de l'hôte définitif. Selon IVASHKIN (14), l'infestation des animaux se fait toujours par la voie buccale.

Il s'agit donc là d'un cycle en tout point comparable à ceux d'Habronema muscae, d'Ha-

(*) Par Parabronema indicum. 
bronema microstomum et de Draschia megastoma $(6,12)$, avec cette restriction que, chez l'Eléphant, en cas de localisation extra-stomacale, on ne peut éliminer totalement l'éventualité de larves circulant dans le sang (5).

Du point de vue taxonomique, il est parfaitement logique d'inclure le genre Parabronema dans la famille des Habronematidae, à proximité des genres Draschia et Habronema. Cette notion qui a été défendue par BAYLIS (3) et par CHABAUD $(7,6)$ n'a pas toujours été admise : pendant longtemps, les Parabronema, sur le simple fait qu'ils étaient pourvus de cordons céphaliques, ont été placés dans la famille des Acuariidae $(1,32)$ ou dans celle des Histiocephalidae (25) ou encore dans celle des Spiruridae (16).

\section{REMERCIEMENTS}

L'auteur tient à remercier vivement Monsieur le Professeur CHABAUD et Madame A. PETTER de l'aide précieuse qu'ils ont apportée à l'élaboration de ce mémoire.

\title{
SUMMARY
}

Parabronema longispiculatum $\mathbf{n}$, sp., a stomach parasite of african elephant (Loxodonta africana Blumenbach, 1797)

\begin{abstract}
Numerous Parabronema were encountered in the stomach of two african Elephants shot near N'Djamena (Fort-Lamy), Chad.

With regard to the nearest species, Parabronema rhodesiense Yorke and Maplestone, 1926, some differences were observed in measurements of oesophagus, tail of female and left spicule of the male which are longer than in this species. The vulva likewise is situated almost immediatly behind the posterior end of the oesophagus.

In consequence, the author considers this Nematode to belong to a distinct species and the name of Parabronema longispiculatum is proposed.
\end{abstract}

He gives some informations on the lesions caused by these parasites.

\section{RESUMEN}

\author{
Parabronema longispiculatum n. sp., parásito estomacal \\ del elefante de Africa (Loxodonta africana Blumenbach, 1797)
}

En el estómago de dos elefantes matados cerca de NDjamena, Repú blica de Chad, se recogieron numerosos Parabronema.

En relación con la especie más próxima, Parabronema rhodesiense Yorke y Maplestone, 1926. se observaron las diferencias siguientes: Jongitud más importante del esófago, de la cola de la hembra y de la espícula izquierda del macho; posición de la vulva en función de la terminación del esófago. EI autor considera que el Parabronema descubierto en Chad pertenece a una nueva espec1e, Parabronema longispiculatum, distinta de la de Yorke y Maplestone.

Da tambien algunos datos sobre las lesiones observadas.

\section{BIBLIOGRAPHIE}

1. BAER (J.G.). Etude critique des Helminthes parasites de l'Okapi. Acta trop., 1950, 7, (2): 164192.

2. BASSON (P. A.), MCCULLY (R. M.), DE VOS (V.), YOUNG (E.), KRUGER (S. P.). Some parasitic and other natural diseases of African Elephant in the Kruger National Park. Onderstepoort J. vet. Res., 1971, 38 (4) : 239-254.

3. BAYLIS (H. A.). A new genus of Nematodes parasitic in Elephants. Parasilology, 1921, 13: 5766.

4. BERGHE VAN DEN (L.), VUYLSTEKE (C.). Contribution à l'étude des parasites de l'Okapi (Deuxième partie). Rev. Zool. Bot. afr., 1937, 29 (4) : 361-369.

5. CHABAUD (A. G.), MOUCHET (J.). A propos d'un Spiruridé Parabronema africanum BAYLIS,
1921, présent dans le cour et le foie d'un Elephant. Remarques sur la filiation des cycles évolutifs entre Spiruridés et Filaires. Bull. Soc. Path. exot., 1956, 49 (2) : 388-397.

6. CHABAUD (A.G.) in: ANDERSON (R.C.), CHABAUD (A.G.), WILLMOTT (S.), C.I.H. Keys to the Nematodes parasites of Vertebrates, Farnham Royal, Commonwealth Agricultural Bureaux, 1975, 3 (2) : 29-58.

7. CHABAUD (A.G.). Ordre des Spirurida in: GRASSE (P.), Traité de zoologie, Paris, Masson et Cie, 1965,4 (3) : 1064-1074.

8. COBBOLD (T.S.). The parasites of Elephants. Trans. Lin. Soc. London, Zool. II, 1882, $4: 223-$ 258.

9. CONDY (J.B.). Observations on internal parasites in Rhodesian Elephant, Loxodonta africana 
Blumenbach, 1797. Proc. Trans. Rhod. Sci. Ass., 1974, 55 (2): 67-99.

10. DAYNES (P.), GRABER (M.). Principales helminthoses des animaux domestiques en Ethiopie. Rev. Elev. Méd. vét. Pays trop., 1974, 27 (3) : 301-306.

11. DOBBERSTEIN (J.). Ueber Sektionsbefunde bei den in zoologischen Därten gehaltenen. Tieren Berl. Tierärztl. Wschr, 1936, 24 : 389-392.

12. EUZEBY (J.). Les maladies vermineuses des animaux domestiques et leurs incidences sur la pathologie humaine. I. Maladies dues aux Némathelminthes, Fasc. I., Paris, Vigot frères, 1961.

13. FITZSIMMONS (W. M.). Parabronema roundi n. Sp. (Nematoda: Spiruridae) from Rhinoceros bicornis in Kenya. J. Helminth., 1962, 36 (1/2): 39-44.

14. IVASHKIN (V. M.). La Parabronématose, une nouvelle helminthose des Ruminants. Epizootologie, prophylaxie, élucidation du cycle évolutif de son agent. Bull. Off. int. Epizoot., 1958, 49 bis, $(1 / 12): 352-360$.

15. IVASHKIN (V.M.). Parabronemiasis in Ruminants. Veterinariya, 1959, 36 (6): 26-8 (en russe).

16. IVASHKIN (V. M.). Nematodes of the subfamily Parabronematinae SKRJABIN, 1941, in the light of morphology and systematics. Trudy gel'mint. Lab., 1960, 10: 94-108 (en russe).

17. KHALIL (M.). A revision of the Nematodes parasites of Elephants, with a description of four new species. Proc. zool. Soc. London, 1922, 15 : 215-219.

18. KHALIL (M.). Une nouvelle espèce de Parabronema du Rhinocéros, Parabronema rhinocerotis n. Sp.; Annls. Parasit. hum. comp., 1927, 5 (1) : $37-40$.

19. LEIPER (R. T.). Helminth parasites obtained from the Okapi at post-mortem. Prac. zool. Soc. London, 1935, 349 : 949.

20. ROUND (M.C.). Check-list of Helminth parasites of african Mammals. Farnham Royal, C.A.B. (Tech. Communication no 38), 113-114.

21. SARWAR (M. M.). On the transference of Para- bronema skrjabini RASSOWSKA, 1924 to Squamanema THIEL, 1925 and its occurrence in sheep and goats in Indo-Pakistan. J. Helminth., 1954, 28 (3/4) : $149-150$.

22. SARWAR (M. M.), SHAIKH (I. Y.). Helminthiasis in an East Pakistan Elephant with a short review of the literature. Ceylon vet. J., 1956, 4 (3): 84.

23. SCHWARTZ (B.), ALICATA (J. E.). Description of two parasitic Nematodes from the Texas Peccary. Proc. U.S. natn. Mus., 1933, 82 (15) : 1-6.

24. SENEVIRATNA (P.), JAYASINGHE (J, B.). Some parasites from the Ceylon Elephant (Ele phas maximus). Ceylon vet. J., 1969, 16 (1/2): 23.

25. SKRJABIN (K. I.). On the rearrangement of the taxonomy of Nematodes of the Acuariidae and Ancyracanthidae. Dokl. Akad. Nauk. S.S.S.R., 1941, 30: 470-473 (en russe).

26. SUTHERLAND (A. K.), O'SULLIVAN (P. J.), OHMAN (A.F.S.). Helminthiasis in an Elephant. Aust. vet. J., 1950, 26 (4) : 88-89.

27. THIEL VAN (P. H.). Deux Nématodes nouveaux d'un singe hurleur du Surinam. Annls. Parasit. hum. comp., 1925, 3 (2) : 171-180.

28. TRONCY (P. M.), GRABER (M.), THAL (J.). Note sur la présence de Parabronema skrjabini Rassowska, 1924 chez les ruminants sauvages d'Afrique. Rev. Elev. Med. vét. Pays trop., 1973, 26 (1) : 93-116.

29. VUYLSTEKE (C.). Nématodes parasites de l'Okapi. Rev. Zool. Bot. afr., 1935, 27 (3/4) : 313-318.

30. VUYLSTEKE (C.). Notes sur les Nématodes parasites de l'Eléphant d'Afrique. Rev. Zool. Bot. Afr., 1953, 48 (3/4) : 213-239.

31. WHESTHUYSEN VAN (O. P.). A monograph of the helminth parasites of the Elephant. Onderstepoort J. vet. Sci. anim. Ind., 1938, 10 (1) : 49-189.

32. YORKE (W.), MAPLESTONE (P. A.). The Nematodes parasites of vertebrates. London, J. and B. Churchill, 1926, 337. 